\title{
Computer simulation of the skin reflectance spectra
}

\author{
I.V. Meglinski ${ }^{a, *}$, S.J. M atcher ${ }^{b}$ \\ a School of Engineering, Cranfield U niversity, Cranfield M K $43 \mathrm{OAL}$, UK \\ ${ }^{b}$ School of Physics, University of Exeter, Exeter EX $44 Q \mathrm{~L}, \mathrm{UK}$
}

\begin{abstract}
The reflectance spectra of the human skin in visible and near-infrared (NIR) spectral region have been calculated using the $M$ onte $C$ arlo technique, and the specular and internal reflection on the medium surface is taken into account. Skin is represented as a complex inhomogeneous multi-layered highly scattering and absorbing medium. The model takes into account variations in spatial distribution of blood, index of blood oxygen saturation, volume fraction of water and chromophores content. The simulation of the skin tissues optical properties and skin reflectance spectra are discussed. Comparison of the results of simulation and in vivo experimental results are given.
\end{abstract}

Keywords: Skin reflectance spectra; M onte Carlo; M ulti-layered medium; Skin tissues optical properties

\section{Introduction}

The in vivo spectral reflectance measurements of human skin can serve as a valuable supplement to standard non-invasive techniques for diagnosing various skin diseases, such as venous ulcers, skin necrosis, interstitial oedema, etc. However, quantified analysis of the reflectance spectra is complicated by the fact that skin has a complex multilayered non-homogeneous structure $[1,2]$ with a

\footnotetext{
* Corresponding author. Tel.: +44-1234-754-767; fax: +441234-750-425

E-mail address: i.meglinski@cranfield.ac.uk (I.V. M eglinski).
}

spatially varying absorption coefficient, mainly determined by melanin pigmentation, oxygen saturation of cutaneous blood, index of erythema, bilirubin, $\beta$-carotine and other chromophores [3].

In most of clinical applications given the measured reflectance spectra, we need to extract the concentrations of various chromophores of interest, in particular oxy- and deoxy-hemoglobin, water and melanin. Various approaches exist but in our initial work we have applied the simplest technique-the modified Beer $-\mathrm{L}$ ambert law. This method attempts to account for the spectral distortions introduced by multiple scattering via a simple linearised equation which relates overall tissue attenuation $-\ln \left(\mathrm{I} / \mathrm{I}_{0}\right)$ to the tissue absorption coefficient $\mu_{\mathrm{a}}$ : 
$\mathrm{A}=-\ln \left(\frac{\mathrm{I}}{\mathrm{I}_{0}}\right)=\mu_{\mathrm{a}} \sigma \rho+\mathrm{G}$

where $\rho$ is the source-detector spacing and $\sigma$ is a scaling factor, the 'differential pathlength factor' which accounts for the path lengthening effect of the random walk experienced by photons as they propagate through the multiple scattering medium. $G$ is an offset term which is, according the Twersky's multiple-scattering theory $[4,5]$, determined mainly by the tissue-probe geometry, or purely by the scattering.

Eq. (1) represents a linear relationship between $-\ln \left(I / I_{0}\right)$ and $\mu_{a}$, then the technique of multilinear regression can be used to estimate the relative concentrations of chromophores in the skin, provided the wavelength dependence of $G$ is known.

Since $G$ is determined by scattering coefficient $\mu_{\mathrm{s}}$, then we will assume that it is dominated by a term of the form $a+b \lambda . \mu_{a}$ is given by the sum of absorption coefficients for each separate chromophore, which in turn are determined by the absolute concentration $\mathrm{C}$ and specific absorption coefficient $\varepsilon$ of each chromophore. Given N chromophores, Eq. (1) can thus be rewritten:

$A=a+b \lambda+\sum_{i=1}^{N} C_{i} \varepsilon_{i}(\lambda)$

By making measurements of $A$ at a minimum of $\mathrm{N}+3$ wavelength, solving Eq. (2) for $a, b$ and $C_{i}{ }^{\prime} s$ becomes an exercise in multi-linear regression which we solve using standard algorithms [6]. Typically we fit all wavelengths from 550 to 770 $\mathrm{nm}$ in $0.7 \mathrm{~nm}$ steps; hence the number of wavelength greatly exceeds $\mathrm{N}$.

It should always be borne in mind however that Eq. (1) is simply an approximation. The modified Beer-Lambert law was originally introduced to analyze near-infrared transmission spectra of brain and muscles and, in particular, was designed to analyze relative changes in tissue oxygenation [7]. D uring such changes $G$ and $\sigma$ (see Eq. (1)) can, to first order, be assumed to remain unchanged so that Eq. (2), in the form:
$\Delta \mathrm{A}(\lambda)=\sum_{\mathrm{i}=1}^{\mathrm{N}} \Delta \mathrm{C}_{\mathrm{i}} \varepsilon_{\mathrm{i}}(\lambda)$

is used to estimate in oxy- and deoxy-hemoglobin concentration from changes in attenuation $\Delta \mathrm{A}$. The equation is strictly only valid in the limit that $\Delta C_{i} \rightarrow 0$, otherwise the fundamentally nonlinear relationship between $\mathrm{A}$ and $\mu_{\mathrm{a}}$ introduces errors. This effect has been studied using the diffusion equation for the specific case of detecting cytochrome-oxidase redox changes in the presence of large changes in hemoglobin absorption [8].

F or this reason, it is one of the general goals of our $\mathrm{M}$ onte Carlo modeling to investigate the validity of the simple multi-linear regression approach. This can be done simply by comparing the actual chromophore concentrations used to perform M onte Carlo calculation with the chromophore concentrations extracted from the simulated reflectance spectrum by Eq. (2).

In this paper we present the method for the skin reflectance spectra simulation, that is done using the $\mathrm{M}$ onte Carlo technique. Different cells structure and blood distribution, variations of chromophores and water content in the skin layers affect their optical properties $[9,10]$, which makes it difficult to define the optical events in a simulation. We simulate the absorption properties of skin layers corresponding to blood, water, melanin and overall chromophores content. Several efforts have been made to simulate the reflectance spectrum of skin relying on a diffusion approximation [11-14]. H owever, when the source-detector separation is 'small' (less than a few millimeters) diffusion approximation becomes invalid, and only the Monte Carlo technique can provide a realistic model of light propagation in biological tissues. The small source-detector separation is often preferred as it yields a shallow spatial location of the subject of study, which are capillary loops in our case. We propose more accurate model for correct prediction of the reflectance spectra measured by 'shallow' fiber-optic reflectance probe. In the paper we simply demonstrate the accuracy of the model by comparing the results of simulation and experimental results made in vivo. 


\section{Reflectance spectra simulation}

\subsection{M ethod}

The skin reflectance spectra simulation is based on a Monte Carlo model developed recently $[15,16]$. In frame of the technique the simulation is performed as a sequential three dimensional tracing of photon packets between scattering events from the point of the radiation entering in medium, to the receiving area, where the photon leaves the medium. The random path that photons move at $\mathrm{j}$ th step is given by:

$\mathrm{I}_{\mathrm{j}}=-\frac{\ln (\xi)}{\mu_{\mathrm{s}}}$

where $\xi$ is a uniformly distributed random number between 0 and 1 . The scattering event is simulated by generating two random angles $\varphi$ and $\theta$ in respect to the Henyey $-\mathrm{G}$ reenstein angular probability density function [17].

Internal reflection on the medium boundary is taken into account allowing the photon packet to split into a reflected and a transmitted part. The statistical weight of the reflected and transmitted parts of the photon packets is attenuated according to the Fresnel's reflection coefficients [18]:
The probability of the photon packet being detected can then be described as follows:

$W=W_{0} \prod_{k=1}^{M} R_{k}\left(\alpha_{i}\right)$

where $\mathrm{W}_{0}$ is the initial weight of the photon packet in the medium, $M$ is the number of photon packet partial reflections on the medium boundary.

The individual trajectory of each detected photon packet is stored in a data file, and then we include the absorption of the medium layers according the microscopic Beer-L ambert law:

$I(\lambda)=\sum_{j}^{N_{p h}} W_{j} \exp \left(-\left.\sum_{i=1}^{K_{j}} \mu_{a i}(\lambda)\right|_{i}\right)$

Here, $W_{j}$ is the final weight of $j$ th photon packet (see Eq. (6)), $K_{j}$ is the total number of scattering events for the $j$ th photon packet, $\mu_{a i}$ and $l_{i}$ are the medium local absorption coefficient and pathlength of the photon packet at ith step, respectively. This approach is convenient for a rapid recalculation of the radiation intensity reflection for a various set of absorption coefficients $\mu_{\mathrm{ai}}(\lambda)$, and their various derivatives for the unchanged source-detector configuration.

The total diffuse reflectance on the medium boundary is defined as the normalized sum of

$R\left(\alpha_{i}\right)=\left\{\begin{array}{lll}\left(\frac{n-n_{0}}{n+n_{0}}\right)^{2}, & \text { if } \quad \alpha_{i}=0^{\circ} \\ \frac{1}{2}\left[\frac{\sin ^{2}\left(\alpha_{i}-\alpha_{t}\right)}{\sin ^{2}\left(\alpha_{i}+\alpha_{t}\right)}+\frac{\tan ^{2}\left(\alpha_{i}-\alpha_{t}\right)}{\tan ^{2}\left(\alpha_{i}+\alpha_{t}\right)}\right], & \text { if } & 0^{\circ}<\alpha_{i}<\sin ^{-1}\left(\frac{n_{0}}{n}\right) \\ 1 & \text { if } & \sin ^{-1}\left(\frac{n_{0}}{n}\right)<\alpha_{i} \leq 90^{\circ}\end{array}\right.$

where $\alpha_{i}$ and $\alpha_{t}$ are the angles of the photon packet incidence on the medium boundary and angle of transmittance, respectively, $n$ is the refractive index of the first layer of the medium and $n_{0}$ represents the refractive index of the ambient medium. statistical weights of the photon packets reaching the detector area:

$$
\mathrm{I} / \mathrm{I}_{0}=\frac{1}{\mathrm{~N}_{\mathrm{ph}} \mathrm{W}_{0}} \sum_{\mathrm{j}}^{\mathrm{N}_{\mathrm{ph}}} \mathrm{W}_{\mathrm{j}} \exp \left(-\sum_{\mathrm{i}=1}^{\mathrm{K}_{\mathrm{i}}} \mu_{\mathrm{ai}}(\lambda) \mathrm{I}_{\mathrm{i}}\right)
$$


Here, $\mathrm{N}_{\mathrm{ph}}$ is the total number of detected photon packets (typically $10^{5}-10^{6}$ ). The simulation of a photon packet is stopped if the photon statistical weight falls bellow 0.0001 , or if a total number of scattering events exceed 10000 .

\subsection{Skin model}

Following earlier work aimed at the skin optical radiation dose modeling $[9,14,19-21]$ we have considered skin as a three-dimensional half-infinite medium divided into seven layers. The first layer corresponds to the layer of desquamating flattened dead cells mainly containing keratin, which is 20 $\mu \mathrm{m}$ thick, and known as the stratum corneum. The second layer, we call Living epidermis, is $80-100$ $\mu \mathrm{m}$ thick and is assumed to contain primarily living cells: a fraction of dehydrated cells, laden cells with keratohyalin granules, columnar cells, and also melanin dust, small melanin granules and melanosoms [1,2]. Given the inhomogeneous distribution of the blood vessels and skin capillaries within the skin [22] we sub-divide the dermis into four different layers, with different blood volumes. These layers are: pappilary dermis (150-200 $\mu \mathrm{m}$ thick), upper blood net dermis (80-100 $\mu \mathrm{m}$ thick), reticular dermis (1400-1600 $\mu \mathrm{m}$ thick), deep blood net dermis (80-120 $\mu \mathrm{m}$ thick). The deepest layer in our model is subcutaneous fat $(6000-6500 \mu \mathrm{m}$ thick).

\subsection{Skin optics simulation}

In the framework of our model we have calculated the absorption coefficients of dermal layers $\mu_{a}^{\text {Layer }}$ taking into account the spatial distribution of blood and water content within the skin, oxygen saturation $S$, and total hemoglobin volume fraction in blood $\gamma$ :

$$
\begin{aligned}
\mu_{\mathrm{a}}^{\text {Layer }}(\lambda)= & (1-\mathrm{S}) \gamma \mathrm{C}_{\text {Blood }} \mu_{\mathrm{a}}^{\mathrm{Hb}}(\lambda)+\mathrm{S} \gamma \mathrm{C}_{\text {Blood }} \mu_{\mathrm{a}}^{\mathrm{HbO}_{2}(\lambda)} \\
& +\left(1-\gamma \mathrm{C}_{\text {Blood }}\right) \mathrm{C}_{\mathrm{H}_{2} \mathrm{O}} \mu_{\mathrm{a}}^{\mathrm{H}_{2} \mathrm{O}}(\lambda) \\
& +\left(1-\gamma \mathrm{C}_{\text {Blood }}\right)\left(1-\mathrm{C}_{\mathrm{H}_{2} \mathrm{O}}\right) \mu_{\mathrm{a}}^{\text {Other }}(\lambda)
\end{aligned}
$$

Here, $\mu_{\mathrm{a}}^{\mathrm{HbO}_{2}}(\lambda), \mu_{\mathrm{a}}^{\mathrm{Hb}}(\lambda), \mu_{\mathrm{a}}^{\mathrm{H}_{2} \mathrm{O}}(\lambda)$ are the absorption coefficients of oxy- and deoxy-hemoglobin and water, respectively, $\mathrm{C}_{\mathrm{Blood}}$ and $\mathrm{C}_{\mathrm{H}_{2} \mathrm{O}}$ are the layer

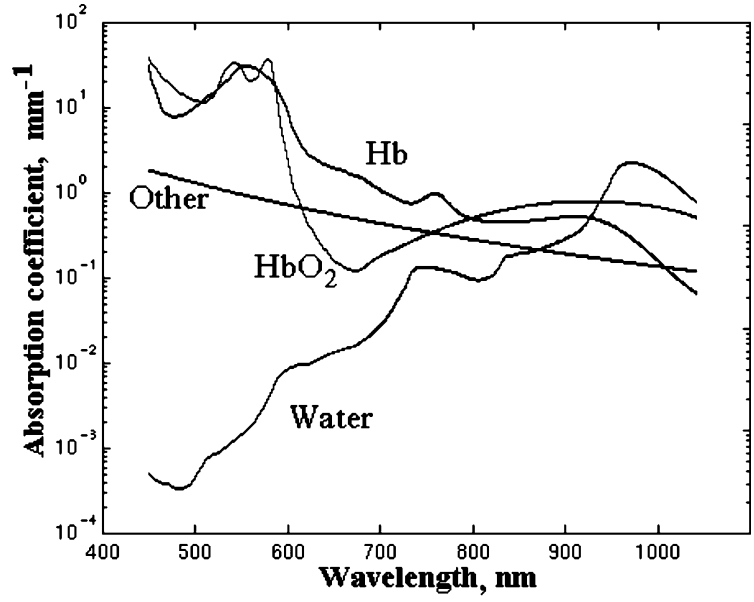

Fig. 1. The absorption coefficients of oxy- $\left(\mathrm{HbO}_{2}\right)$, deoxy- $(\mathrm{Hb})$ hemoglobin, water (W ater) and hemoglobin -water free tissues (Other) in visible and near infrared (N IR) range of spectra.

volume fractions of blood and water contents, and $\mu_{\mathrm{a}}^{\text {Other }}$ is the absorption coefficient of the hemoglobin-water free tissue, defined as [23]:

$\mu_{\mathrm{a}}^{\text {Other }}(\lambda)=7.84 \times 10^{7} \times \lambda^{-3.255}$

We have calculated $\gamma$ assuming that hemoglobin is contained in the erythrocytes only, i.e.

$\gamma=\mathrm{F}_{\mathrm{Hb}} \mathrm{F}_{\mathrm{RBC}} \mathrm{H} \mathrm{t}$

where $\mathrm{Ht}$ is the hemotocrit, $\mathrm{F}_{\mathrm{RBC}}$ is the volume fraction of erythrocytes in the total volume of all blood cells, $\mathrm{F}_{\mathrm{Hb}}$ is the volume fraction of hemoglobin in erythrocytes.

The absorption coefficients: $\mu_{\mathrm{a}}^{\mathrm{Hb}}(\lambda), \mu_{\mathrm{a}}^{\mathrm{HbO}_{2}(\lambda),}$ $\mu_{\mathrm{a}}^{\mathrm{H}_{2} \mathrm{O}}(\lambda)$ and $\mu_{\mathrm{a}}^{\text {Other }}(\lambda)$ for the optical/N IR $(400-$ $1100 \mathrm{~nm}$ ) range of spectrum are presented in Fig.

Table 1

Optical properties of the model of the human skin in the visible range of the spectrum $(\lambda=633 \mathrm{~nm})$

\begin{tabular}{llcll}
\hline $\mathrm{k}$ & N ame of layer & $\mu_{\mathrm{s}}\left(\mathrm{mm}^{-1}\right)$ & $\mathrm{g}$ & $\mathrm{n}$ \\
\hline 1 & Stratum corneum & 100 & 0.86 & 1.5 \\
2 & Living epidermis & 45 & 0.8 & 1.34 \\
3 & Papillary dermis & 30 & 0.9 & 1.4 \\
4 & U pper blood net dermis & 35 & 0.95 & 1.39 \\
5 & R eticular dermis & 25 & 0.8 & 1.4 \\
6 & Deep blood net dermis & 30 & 0.95 & 1.38 \\
7 & Subcutaneous fat & 5 & 0.75 & 1.44 \\
\hline
\end{tabular}


1. The absorption factors for oxy- and deoxyhemoglobin and water are re-calculated from their spectra of extinction coefficients $[24,25]$ with respect to their relative concentrations. In terms of optical density (1) measurements, absorption of water is significantly low relative to the absorption of oxy- and deoxy-hemoglobin. In a normal human skin, however, the content of water molecules is approximately $3 \times 10^{5}$ versus one molecule of hemoglobin [26]. Other skin layers optical properties used in the simulation: scattering coefficients $\mu_{\mathrm{s}}$, anisotropy factors $\mathrm{g}$ and refractive indices $\mathrm{n}$ are represented in Table 1 . These data are collected from the literature: the scattering coefficients and anisotropy factors are taken from $[9,10,27,28]$, and refractive indices from $[9,29,30]$. The values quoted at the wavelength $\lambda=632 \mathrm{~nm}$. The refractive index of the ambient medium is taken to be $n_{0}=1$.

\section{Results and discussions}

The absorption coefficients calculated by Eq. (9) for the dermal layers are presented in $\mathrm{Fig} .2$. Parameters $\mathrm{C}_{\mathrm{Blood}}, \mathrm{C}_{\mathrm{H}_{2} \mathrm{O}}, \mathrm{S}, \mathrm{Ht}, \mathrm{F}_{\mathrm{Hb}}, \mathrm{F}_{\mathrm{RBC}}$ used for the calculation are presented in Table 2. These data are collected from a range of literature

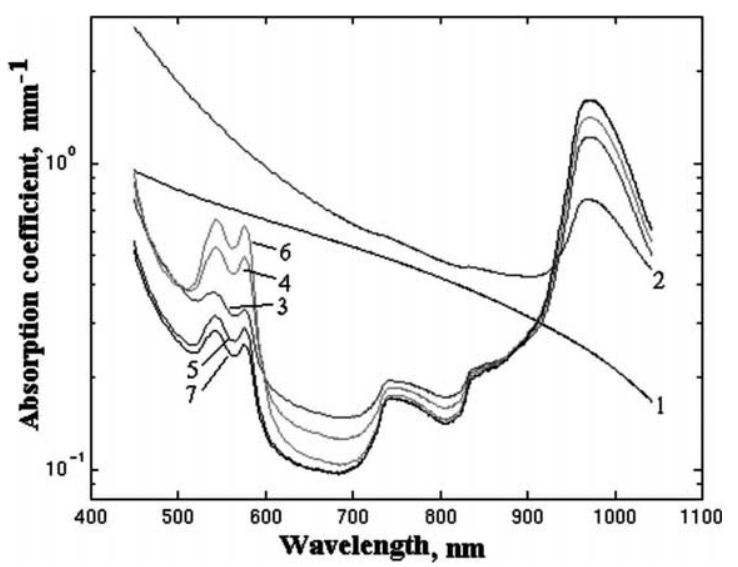

Fig. 2. Simulated absorption coefficients of skin layers: (1) stratum corneum; (2) living epidermis; (3) pappilary dermis; (4) upper blood net dermis; (5) reticular dermis; (6) deep blood net dermis; (7) subcutaneous fat.
$[1,2,22,26,31-35]$, that, we believe, is corresponded to skin at normal stage.

A bsorption coefficients for the blood free layers of skin, i.e. for stratum corneum and living epidermis, are calculated similar to what is proposed in [10]. We assume that $\mu_{\mathrm{a}}(\lambda)$ of stratum corneum is described as:

$$
\begin{aligned}
\mu_{\mathrm{a}}^{\text {Stratum }}(\lambda)= & \left(0.1-8.3 \times 10^{-4} \times \lambda\right)+0.125 \\
& \times \mu_{\mathrm{a}}^{\text {Other }}(\lambda)
\end{aligned}
$$

and for living epidermis water content and the experimental data $[36,37]$ are taken into account:

$$
\begin{aligned}
& \mu_{\mathrm{a}}^{\text {living }_{\text {epidermis }}}(\lambda) \\
& =\left(0.5 \times 10^{10} \times \lambda^{-3.33}\right)\left(1-\mathrm{C}_{\mathrm{H}_{2} \mathrm{O}}\right) \\
& \quad+\mathrm{C}_{\mathrm{H}_{2} \mathrm{O}} \mu_{\mathrm{a}}^{\mathrm{H}_{2} \mathrm{O}}(\lambda)
\end{aligned}
$$

The results of the simulation (see $\mathrm{F}$ ig. 2) are well agreed with the experimental results for Caucasian skin [38], and have showed that absorption of the visible/NIR radiation in stratum corneum and living epidermis decreases uniformly with wavelength from 450 to $900 \mathrm{~nm}$. The peaks at 970-980 $\mathrm{nm}$ featured in all layers, except stratum corneum, are produced by the water absorption (see Fig. 1 and Table 2).

As one can see from Fig. 2 in the blood containing layers (i.e. pappilary dermis, upper blood net dermis, reticular dermis, deep blood net dermis and subcutaneous fat) the absorption of oxy- and deoxy-hemoglobin dominates for the wavelength shorter than $600 \mathrm{~nm}$ (see Fig. 1 for comparison). In the range of $600-800 \mathrm{~nm}$ the absorption is minimum (see $\mathrm{Fig}$. 2).

$U$ sing the optical properties we simulate the skin reflectance spectra by the $\mathrm{M}$ onte Carlo technique described above. We have found that the results of simulation are remarkably similar to the experimental results of skin spectra measurements in visible (450-600 nm) range of spectrum (Fig. 3).

The measured reflectance spectra of skin are obtained by the spectrophotometer system described in detail earlier $[39,40]$. In short, the skin reflectance spectra are collected from the surface of the tissue with a pair of optical fibers separated at fixed distance $(400 \mu \mathrm{m})$. The detected fiber collects light within a range of angles defined by 
Table 2

The parameters used in the calculation of the absorption coefficients of the blood contented layers of the skin

\begin{tabular}{|c|c|c|c|c|c|c|c|}
\hline k & $\mathrm{N}$ ame of layer & $\mathrm{C}_{\text {Blood }}$ & $\mathrm{S}$ & $\mathrm{Ht}$ & $\mathrm{F}_{\mathrm{Hb}}$ & $\mathrm{F}_{\mathrm{RBC}}$ & $\mathrm{C}_{\mathrm{H}_{2} \mathrm{O}}$ \\
\hline 1 & Stratum corneum & 0 & 0 & 0 & 0 & 0 & 0.05 \\
\hline 2 & Living epidermis & 0 & 0 & 0 & 0 & 0 & 0.2 \\
\hline 3 & Papillary dermis & 0.04 & 0.6 & 0.45 & 0.99 & 0.25 & 0.5 \\
\hline 4 & U pper blood net dermis & 0.3 & 0.6 & 0.45 & 0.99 & 0.25 & 0.6 \\
\hline 5 & R eticular dermis & 0.04 & 0.6 & 0.45 & 0.99 & 0.25 & 0.7 \\
\hline 6 & D eep blood net dermis & 0.1 & 0.6 & 0.45 & 0.99 & 0.25 & 0.7 \\
\hline 7 & Subcutaneous fat & 0.05 & 0.6 & 0.45 & 0.99 & 0.25 & 0.7 \\
\hline
\end{tabular}

$C_{B l o o d}$ derived from R efs. $[1,2,22,26,31-34]$ and personnel communication with Professor $A$. Shore, $C_{H_{2}}$ o evaluated from R efs. [26,35], $\mathrm{S}, \mathrm{H}$ t, $\mathrm{F}_{H b}, \mathrm{~F}_{\mathrm{RBC}}$ derived from R efs. [22,26,32] and personnel communication with Professor $A$. Shore.

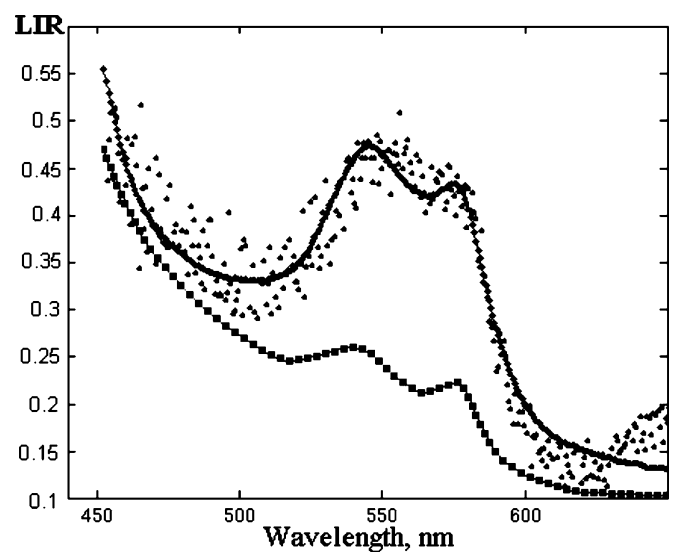

Fig. 3. The skin reflectance spectra: rhombuses and dots represent the results of simulated and measured reflectance spectra, respectively. Squares are the results of skin spectrum simulation with the $40 \%$ reduction of blood volume in the first two dermal layers (i.e. in pappilary dermis and upper blood net dermis). LIR is defined as the logarithm to the base 10 of the inverse reflectance $\left(R=I / I_{0}\right)$, i.e. $\log _{10}(1 / R)$ or $\log _{10}(1 / W)$, respectively.

the numerical aperture. A quartz halogen lamp is used as an optical/NIR source of light, and a spectrograph/monochromator with a CCD camera is used for high speed spectra measurements, where one dimension is responsible for wavel ength and the other for the intensity of detected scattering light. Our choice of source-detector optical fibers spacing is based on the results of spatial sensitivity study reported recently $[15,16]$. In these studies we showed that for $400 \mu \mathrm{m}$ sourcedetector spacing the main part of detected signal becomes localized in the topical skin layers includ- ing pappilary dermis and upper blood net dermis. The results of reflectance spectra simulation in case of $40 \%$ blood content reduction in topical dermal layers (i.e. pappilary dermis and upper blood net dermis) (see Fig. 3) well illustrate what happens if the absorption properties of the skin are changed. A $n$ increase of the blood content in the upper layers will tend to reduce the overall path length in that layers, conversely a decrease in blood content will lengthen the path length in it. $\mathrm{H}$ owever, we believe that amount of blood used in our simulation (see Table 2) was reliably estimated for the true blood content in the skin tissues at normal stage. These results are agreed with the results of independent study of the skin color variations [41].

We have presented the visible spectral region only, as it was best fitted (see Fig. 3). The difference in the results of simulation and experimental data (see Fig. 3) could be explained by our choice of the optical properties of the skin tissues for the simulation, and possible experimental errors. In the model we did not include the influence of various pigments as melanin, bilirubin, NADH , $\beta$-carotine and other chromophores [3], temperature dependence and some physiological parameters. In frame of the proposed model shape of the simulated skin spectra is determined by the combination of spectra of oxy- and deoxyhemoglobin, water and summarized absorption of other chromophores. We simulated the skin reflection spectra assuming the wavelength independence of the skin layers scattering ( $\mu_{\mathrm{s}}$ and $\mathrm{g}$ are constant, see Table 1 ). Whereas, in reality the 
scattering of skin tissues tends to monotonously decrease from 450 to $1100 \mathrm{~nm}[28,29]$, and the difference in scattering at 450-600 and 700-1100 $\mathrm{nm}$ ranges is significant. $\mathrm{N}$ evertheless, the results of simulation show that in the range of spectra where the scattering of skin tissues is changed slightly, it is possible to compare the experimental and modeling results quantitatively.

\section{Summary}

The reflectance spectra of human skin have been studied to support the theoretical model of skin tissues optical properties. The reflectance spectra of human skin simulated by the Monte Carlo technique and measured in vivo are in good agreement with one another. The modeling results are a brief survey of the potential of the computer simulation of skin reflectance spectra. Presented approach can be considered for use in studies of oxygen saturation of blood, skin chromophores, pigmentation, erythema and skin color changing. The model fitting based on the multi-linear regression analysis can give us quantitative amount of skin chromophores of the interest. Including in the model the wavelength dependence of $G$ (see Eq. (1)) it is possible to extend the boundaries of the spectral region for the quantitative model fitting in NIR spectral area in the future.

\section{Acknowledgements}

This work was supported in part by EPSR C grant GR/L 89433. We thank Professor Angela Shore and Doctor Paul Collier for useful and helpful discussions concerning human skin structure and its properties.

\section{References}

[1] K.S. Stenn, The skin, in: L. Weiss (Ed.), Cell and Tissue Biology, U rban \& Shwarzenberg, Baltimore, MD, 1988, pp. $541-572$.
[2] G.F. Odland, Structure of the skin, in: L.A. Goldsmith (Ed.), Physiology, Biochemestry and M olecular Biology of the Skin, vol. I, Oxford U niversity Press, Oxford, 1991, pp. 3-62.

[3] A.R. Y oung, Chromophores in human skin, Phys. M ed. Biol. 42 (1997) 789-802.

[4] V. Twersky, Multiple scattering of waves and optical phenomena, JOSA 52 (1962) 145-171.

[5] V. Twersky, A bsorption and multiple scattering by biological suspensions, J OSA 60 (1970) 1084-1093.

[6] W.H. Press, S.A. Teukolsky, W.T. Vetterling, B.P. Flannery, Numerical Recipes in $\mathrm{C}$, 2nd ed., Cambridge U niversity Press, Cambridge, 1992.

[7] D.T. Delpy, M. Cope, P. van der Zee, S.R. A rridge, S. W ray, J. Wyatt, Estimation of optical pathlength through tissue from direct time of flight measurement, Phys. M ed. Biol. 33 (1988) 1433-1442.

[8] S.J. M atcher, C.E. Elwell, C.E. Cooper, M. Cope, D.T. D elpy, Performance comparison of several published tissue near-infrared spectroscopy algorithms, A nal. Biochem. 227 (1995) 54-68.

[9] V.V. Tuchin, Tissue Optics: Light Scattering M ethods and Instruments for M edical Diagnosis, SPIE - The International Society for Optical Engineering, Washington, DC, 2000.

[10] S.L. Jacques, Origins of tissue optical properties in the UVA, visible and NIR regions, in: R.R. Alfano, J.G. F ujimoto (Eds.), A dvances in Optical I maging and Photon M igration, vol. 2, Optical Society of America (OSA), W ashington, DC, 1996, pp. 364-370.

[11] S. Wan, R.R . A nderson, J.H. Parrish, A nalytical modeling for the optical properties of the skin with in vitro and in vivo applications, Photochem. Photobiol. 34 (1981) 493499.

[12] P.H. Andersen, P. Bjerring, Noninvasive computerized analyses of skin chromophores in vivo by reflectance spectroscopy, Photodermatol. Photoimmunol. Photomed. 7 (1990) 249-257.

[13] L.T. N orvang, E.J . F iskerstrand, K. K oenig, B. Bakken, D. Grini, O. Standahl, T.E. M ilner, M.W. Berns, J.S. $\mathrm{N}$ elson, L.O. Svaasand, Comparison between reflectance spectra obtained with an integrating sphere and a fiberoptic collection system, in: G.P. D elacretaz, R.W. Steiner, L.O. Svaasand, H.J. A lbrecht, T.H. M eier (Eds.), LaserTissue Interaction and Tissue Optics, Proc. SPIE, vol. 2624, 1995, pp. 155-164.

[14] G. Kumar, J.M. Schmitt, Optimum wavelength for measurement of blood hemoglobin content tissue hydration by NIR spectroscopy, in: D.L. Farkas, R.C. L eif, A.V. Priezzhev, T. A sakura, B.J . Tromberg (Eds.), Optical Diagnostics of $L$ iving Cells and Biofluids, Proc. SPIE, vol. 2678, 1996, pp. 442-453.

[15] I.V. M eglinskii, S.J. M atcher, The analysis of spatial distribution of the detector depth sensitivity in multilayered inhomogeneous highly scattering and absorbing medium by the $\mathrm{M}$ onte $\mathrm{C}$ arlo technique, $\mathrm{O}$ pt. Spectrosc. 91 (2001) 654-659. 
[16] I.V. M eglinsky, S.J. M atcher, Modeling the sampling volume for the skin blood oxygenation measurements, M ed. Biol. Eng. Comput. 39 (2001) 44-50.

[17] L.G. Henyey, J.L. Greenstein, Diffuse radiation in the galaxy, A strophys. J. 93 (1941) 70-83.

[18] M. Born, E. Wolf, Principles of Optics: Electromagnetic Theory of Propagation, Interference and Diffraction of L ight, 6th ed., Pergamon Press, N ew Y ork, 1986.

[19] M. K eijzer, S.L. Jaques, S.A. Prahl, A.J. Welch, Light distribution in artery tissue: M onte Carlo simulation for finite-diameter laser beams, Lasers Surg. M ed. 9 (1989) $148-154$

[20] I.V. Y aroslavsky, V.V. Tuchin, Light propagation in multilayer scattering media: modeling by the M onte Carlo method, Opt. Spectrosc. 72 (1992) 505-509.

[21] S.L. J acques, L. W ang, L .-Q. Zheng, M CM L -M onte Carlo modeling of photon transport in multi-layered tissues, Comput. M ethods Programs Biomed. 47 (1995) 131-146.

[22] T.J. Ryan, Cutaneous circulation, in: L.A. Goldsmith (Ed.), Physiology, Biochemistry and Molecular Biology of the Skin, vol. II, Oxford U niversity Press, Oxford, 1991, pp. 1019-1084.

[23] I.S. Saidi, Transcutaneous optical measurement of hyperbilirubinemia in neonates, PhD thesis, Rice U niversity, H ouston, TX, 1992.

[24] W.G. Zijlstra, A. Buursma, W.P. M eeuwsen-van der Roest, Absorption spectra of human fetal and adult oxyhemoglobin, de-oxyhemoglobin, carboxyhemoglobin, and methemoglobin, Clin. Chem. 37 (1991) 1633-1638.

[25] G.M. Hale, M.R . Querry, Optical costants of water in the $200-\mathrm{nm}$ to $200-\mu \mathrm{m}$ wavelength region, A ppl. Opt. 12 (1973) $555-653$.

[26] G. Chapman, The Body Fluids and Their Functions, Edward A rnold, London, 1980.

[27] C.R. Simpson, M. K ohl, M. Essenpreis, M. Cope, N earinfrared optical properties of ex vivo human skin and subcutaneous tissues measured using the M onte Carlo inversion technique, Phys. Med. Biol. 43 (1998) 24652478.

[28] R.M .P. Doornbos, R. Lang, M.C. A alders, F.M. Cross, H.J.C.M . Sterenborg, The determination of in vivo human tissue optical properties and absolute chromophore concentrations using spatially resolved steady-state diffuse reflectance spectroscopy, Phys. M ed. Biol. 44 (1999) 967981.

[29] S. G onzalez, M. R ajadhyaksha, R.R. A nderson, N oninvasive (real-time) imaging of histologic margin of a proliferative skin lesion in vivo, Int. Invest. Dermat. 111 (1998) 538-539.
[30] G.J . Tearney, M .E. Brezinski, J.F. Southern, B.E. Bouma, M.R. H ee, J.G. F ujimoto, D etermination of the refractive index of highly scattering human tissue by optical coherence tomography, Opt. Lett. 20 (1995) 2258-2260.

[31] R. Bull, G. A nsell, A.W.B. Stanton, J.R. Levick, P.S. M ortimer, Normal cutaneous microcirculation in gaiter zone (ulcer-susceptible skin) versus nearby regions in healthy young adults, Int. J. M icrocirc. 15 (1995) 65-74.

[32] D. M acN . Surgenor (Ed.), The R ed Blood Cells, A cademic Press, N ew Y ork, 1975.

[33] A. I keda, N . U meda, K. T suda, S. Ohta, Scanning electron microscopy of the capillary loops in the dermal papillae of the hand in primates, including man, J. Electron M icrosc. Tech. 19 (1991) 419-28.

[34] A.J. Jaap, A.C. Shore, A.J. Stockman, J.E. Tooke, Skin capillary density in subject with impaired glucose tolerance and patients with type 2 diabetes, D iabet. M ed. 13 (1996) $160-164$.

[35] R.0. Potts, Stratum corneum hydration: experimental techniques and interpretations of results, J. Soc. Cosmet. Chem. 37 (1985) 9-33.

[36] R.R. Anderson, J. Hu, J.A. Parrish, Optical radiation transfer in the human skin and applications in in vivo remittance spectroscopy, in: R. M arks, P.A. Payne (Eds.), Bioengineering and the Skin, M TP Press, Boston, MA, 1979, pp. 253-265.

[37] J.W. F eather, J.B. Dawson, D.J . Barker, J .A. Cotterill, A theoretical and experimental study of the optical properties of in vivo skin, in: R. Marks, P.A. Payne (Eds.), Bioengineering and the Skin, MTP Press, Boston, MA, 1979, pp. 275-281.

[38] R. M archesini, C. Clemente, E. Pignoli, M. Brambilla, Optical properties of in vivo epidermis and their possible relationship with optical properties of in vivo skin, J. Photochem. Photobiol. B: Biol. 16 (1992) 127-140.

[39] M. Cope, D.T. D elpy, J.S. Wyatt, S.C. Wray, E.O.R. Reynolds, A CCD spectrometer to quantitate the concentration of chromophores in living tissue utilising the water absorption peak of water at $975 \mathrm{~nm}, \mathrm{Adv}$. Exp. M ed. Biol. 247 (1989) 33-41.

[40] M. Osawa, S. N iwa, A portable diffuse reflectance spectrophotometer for rapid and automatic measurement of tissue, M eas. Sci. Technol. 4 (1993) 668-676.

[41] W. Verkruysse, G.W. Lucassen, M.J.C. van Gemert, Simulation of color of port wine stain skin and its dependence on skin variables, Lasers Surg. M ed. 25 (1999) 131-139. 